OPEN ACCESS

Edited by:

Xingchao Shentu,

Zhejiang University, China

Reviewed by:

Peng Zhou,

Parkway Health, China

Xu Chen,

Shanghai Aier Eye Hospital, China

${ }^{*}$ Correspondence:

Xingtao Zhou

doctzhouxingtao@163.com

${ }^{\dagger}$ These authors have contributed equally to this work and share first

authorship

Specialty section:

This article was submitted to

Ophthalmology,

a section of the journal

Frontiers in Medicine

Received: 26 September 2020 Accepted: 05 January 2021

Published: 27 January 2021

Citation:

Zhao J, Zhao J, Yang W, Miao H, Niu L, Shang J, Wang $X$ and Zhou $X$ (2021) Peripheral Anterior Chamber

Depth and Angle Measurements Using Pentacam After Implantation of

Toric and Non-toric Implantable Collamer Lenses.

Front. Med. 8:610590.

doi: 10.3389/fmed.2021.610590

\section{Peripheral Anterior Chamber Depth and Angle Measurements Using Pentacam After Implantation of Toric and Non-toric Implantable Collamer Lenses}

\author{
Jiao Zhao ${ }^{1+}$, Jing Zhao ${ }^{2,3,4 t}$, Wen Yang ${ }^{5}$, Huamao Miao ${ }^{2,3,4}$, Lingling $\mathrm{Niu}^{2,3,4}$, \\ Jianmin Shang ${ }^{2,3,4}$, Xiaoying Wang ${ }^{2,3,4}$ and Xingtao Zhou ${ }^{2,3,4 *}$ \\ ${ }^{1}$ Department of Ophthalmology, People's Hospital of Leshan, Leshan, China, ${ }^{2}$ Eye Institute and Department of \\ Ophthalmology, Eye, Ear, Nose and Throat Hospital, Fudan University, Shanghai, China, ${ }^{3}$ National Health Center Key \\ Laboratory of Myopia (Fudan University), Key Laboratory of Myopia, Chinese Academy of Medical Sciences, Shanghai, \\ China, ${ }^{4}$ Shanghai Research Center of Ophthalmology and Optometry, Shanghai, China, ${ }^{5}$ Department of Ophthalmology, The \\ Third People's Hospital of Chengdu, The Affiliated Hospital of Southwest Jiaotong University, Chengdu, China
}

Purpose: To evaluate the characteristics of peripheral anterior chamber measurements by Pentacam after posterior implantable collamer lenses (ICL) and toric ICL (TICL) with central hole (V4c) implantation.

Methods: Prospective, non-randomized consecutive case series. Forty-six patients undergoing ICL implantation in one eye (Group A) and identically sized TICL in the contralateral eye (Group B) in the Refractive Surgery Center of Eye and ENT Hospital of Fudan University were prospectively included. According to ICL/TICL size, these eyes were further divided into four subgroups. Peripheral anterior chamber depth (PACD) and angle (ACA) in nasal and temporal sides were measured using Pentacam pre-operatively and 12-month post-operatively.

Results: The safety indices were $1.34 \pm 0.32$ and $1.25 \pm 0.16$ and the efficacy indices were $1.20 \pm 0.24$ and $1.19 \pm 0.19$ for ICL and TICL groups, respectively. There was no significant difference in pre-operative PACD or ACA between the two groups. Post-operative PACD and ACA were significantly lower than pre-operative values. Variations of PACD and ACA of TICL group were significantly larger than those of ICL group. The change of ACA for $13.2 \mathrm{~mm}$ lenses was significantly larger than that of $12.6 \mathrm{~mm}$ lenses. Pre-operative CACD and vault were significantly associated with post-operative PACD, while pre-operative ACA and vault were significantly associated with post-operative ACA.

Conclusions: Variations of PACD and ACA were greater in eyes after TICL (V4C) implantation compared with identically sized ICL (V4c) implantation and with larger size than smaller size lens implantation. Pre-operative anterior chamber structure and vault affect post-operative PACD and ACA.

Keywords: toric implantable collamer lens V4c, peripheral anterior chamber depth, anterior chamber angle, Pentacam, myopia, astigmatism 


\section{INTRODUCTION}

Implantation of the implantable collamer lens (ICL)/ toric ICL (TICL) (V4c) with a central port is preferred over corneal refractive surgery by refractive surgeons and patients for its reversibility and excellent visual quality (1-3). ICL is positioned in the ciliary sulcus and protrudes forward to form a vault, resulting in post-operative narrowing of the anterior chamber angle (ACA) width and decreasing the central anterior chamber depth (CACD) (4).

ACA is the key anatomic parameter determining the risk for primary angle closure glaucoma (PACG) (5). Peripheral anterior synechiae and peripheral angle closure (PAC) become significant possibilities when drainage angle is $\leqq$ grade $2\left(\sim 20^{\circ}\right)(6,7)$. In addition to ACA, peripheral anterior chamber depth (PACD) shows good sensitivity for detecting eyes at risk for angle closure (8). Extremely shallow anterior chamber depth (ACD) or narrow ACA leads to the possibility of angle closure glaucoma (ACG) and extraction of the ICL/TICL (9, 10). For these reasons, prediction and monitoring of post-operative ACA and PACD in the long term are essential to improve the safety of ICL/TICL (V4c) implantation.

Biometric studies have demonstrated that ACD significantly correlates with PAC and PACG (11-13). The Pentacam allows for quantitative measurements of the corneal topography, corneal thickness, ACA, ACD at any point, and anterior chamber volume (ACV). Pentacam has been used to screen eyes suspected of having PAC $(13,14)$. In addition, White-to-white (WTW) distance and CACD measured by Pentacam are crucial parameters for sizing ICL/TICL (V4c). Nevertheless, there was only one report on ACA measurement using Pentacam after ICL/TICL (V4c) implantation (15). Furthermore, there have been no comparative specialized studies on PACD or ACA after ICL and TICL (V4c) implantation.

Therefore, in the present study, we performed a prospective, non-randomized contralateral case comparison study to explore the characteristics of PACD and ACA after implantation of ICL/TICL (V4c) with various sizes. Factors that affect postoperative PACD and ACA were also analyzed.

\section{PATIENTS AND METHODS}

\section{Patients}

This was a prospective, non-randomized consecutive case series study. Patients who underwent routine preoperative examinations for ICL/TICL implantation and subsequent ICL (V4c) implantation in one eye (Group A) and an identically-sized TICL (V4c) implantation in the contralateral eye (Group B) between August 2018 and June 2019 were included.

TICL was selected if astigmatic diopter was beyond 0.75 $\mathrm{D}$, or the percentage of astigmatic diopter to spherical diopter was higher than $10 \%$, or the CDVA could be achieved beyond 2 lines if the astigmatic diopter was corrected by TICL. Regardless of whether TICL or ICL was selected, thorough examinations were conducted, and approval was obtained from all patients.

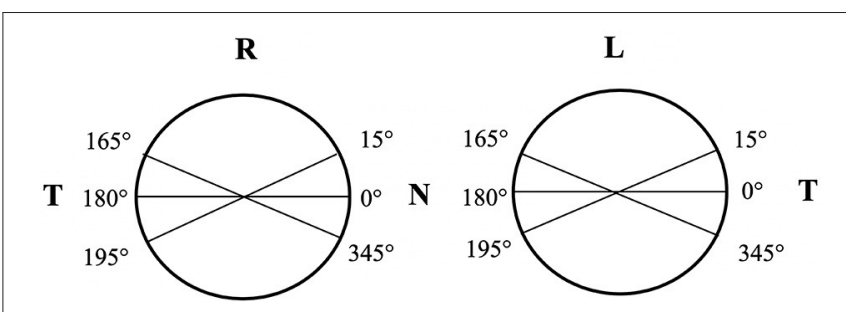

FIGURE 1 | A schematic diagram for calculation of PACD/ACA in nasal and temporal sides of right and left eyes. Arithmetical mean of PACD and ACA at 0 , $15,345^{\circ}$ meridians was considered as the value of the nasal side (NPACD/NACA) in the right eye and temporal side (TPACD/TACA) of the left eye. The arithmetical mean of PACD and ACA at 180, 165, $195^{\circ}$ meridians was considered as TPACD/TACA of the right eye and NPACD/NACA of the left eye.

Groups $A$ and $B$ were further divided into four subgroups: $A_{1}$ (12.6 mm ICL), $\mathrm{A}_{2}$ (13.2 $\mathrm{mm} \mathrm{ICL),} \mathrm{B}_{1}$ (12.6 mm TICL), and $\mathrm{B}_{2}$ (13.2 $\mathrm{mm}$ TICL), based on the size of the ICL/TICL implanted.

This study was approved by the ethics committee and followed the tenets of the Declaration of Helsinki. Written informed consent was obtained from each patient after explanation of the nature and possible consequences of the study.

\section{Examinations}

Pre-operative routine ophthalmic examinations were performed as follows: (1) uncorrected distance visual acuity (UDVA), subjective refraction, corrected distance visual acuity (CDVA), intraocular pressure (IOP) measured using a tonometer (Canon Full Auto Tonometer TX-F; Canon, Tokyo, Japan), slit-lamp examination, axial length, fundus examination, endothelial cell density (ECD) (SP. 2000P; Topcon, Tokyo, Japan) were completed; and (2) horizontal sulcus-to-sulcus (STS) distance was measured using ultrasound biomicroscopy (UBM) (BME300, MEDA, Tianjin, China). Eyes with ciliary or iris cysts were noted.

For Pentacam (OCULUS Optikgeräte GmbH, Wetzlar, Germany) examinations: PACD (defined as the ACD at $4 \mathrm{~mm}$ from the corneal apex) at 6 points and ACA along the 0 , $15,165,180,195$, and $345^{\circ}$ meridians were measured in each eye. The arithmetical means of PACD and ACA at 0 , 15 , and $345^{\circ}$ meridians were considered the values of the nasal side (NPACD/NACA) in the right eye and temporal side (TPACD/TACA) of the left eye. The arithmetical means of PACD and ACA at 180,165 , and $195^{\circ}$ meridians were considered the TPACD/TACA of the right eye and the NPACD/NACA of the left eye (Figure 1). WTW, central corneal thickness, flat keratometry (Kf), steep keratometry (Ks), CACD, pupil diameter (PD), and clear lens rise defined as the distance between the anterior pole of crystalline lens and the horizontal iris plane were also recorded.

\section{ICL/TICL (V4c) Implantation}

ICL/TICL sizing was based primarily on WTW and CACD measurements with Pentacam HR, as recommended by the Staar surgical calculator (http://www.staarvision.com).

All surgeries were performed by two experienced surgeons using the same technique. Binocular procedures were conducted 
TABLE 1 | Pre-operative patient demographic data in group A, B and subgroups.

\begin{tabular}{|c|c|c|c|c|c|c|c|c|}
\hline & \multicolumn{2}{|c|}{ Sex } & \multicolumn{2}{|c|}{ Eye } & \multirow[t]{2}{*}{ Age (years) } & \multirow[t]{2}{*}{ Spherical (D) } & \multirow[t]{2}{*}{ Cylindrical (D) } & \multirow[t]{2}{*}{ SE (D) } \\
\hline & Male & Female & Right & Left & & & & \\
\hline Group A (ICL) & 18 & 28 & 26 & 20 & $25.78 \pm 4.09$ & $-8.97 \pm 2.72$ & $-0.14 \pm 0.22$ & $-9.05 \pm 2.70$ \\
\hline Group A1 (12.6 mm) & 9 & 15 & 14 & 10 & $26.58 \pm 4.78$ & $-8.92 \pm 2.72$ & $-0.17 \pm 0.23$ & $-9.02 \pm 2.70$ \\
\hline Group A2 (13.2 mm) & 9 & 13 & 12 & 10 & $24.91 \pm 0.96$ & $-8.64 \pm 2.50$ & $-0.09 \pm 0.18$ & $-8.70 \pm 2.48$ \\
\hline Group B (TICL) & 18 & 28 & 20 & 26 & $25.78 \pm 4.09$ & $-8.51 \pm 3.08^{\star}$ & $-1.26 \pm 0.38^{\star}$ & $-9.14 \pm 3.09$ \\
\hline Group B1 (12.6 mm) & 9 & 15 & 10 & 14 & $26.58 \pm 4.78$ & $-8.34 \pm 3.13$ & $-1.14 \pm 0.27$ & $-8.91 \pm 3.12$ \\
\hline Group B2 (13.2 mm) & 9 & 13 & 10 & 12 & $24.91 \pm 0.96$ & $-8.35 \pm 2.64$ & $-1.39 \pm 0.48$ & $-9.05 \pm 2.68$ \\
\hline
\end{tabular}

$D$, Diopter; SE, Spherical equivalence.

${ }^{*} P<0.05:$ Group A vs. Group B.

successively, and the right eye was operated on first. Surgical procedures were as previously described (16). Eyes of Group A were implanted with ICL (V4c) in the horizontal axis while eyes of Group B were implanted with TICL (V4c) with rotation of axis within 13 degrees $\left(4.78 \pm 3.02^{\circ}\right.$, range: $\left.0-13^{\circ}\right)$.

\section{Follow-Up}

The patients were followed up for $12.13 \pm 4.28$ months (range: 9-17 months). Follow-up examinations included assessments of UDVA, CDVA, refractive power, ECD, IOP, PACD, ACA, and vault [determined as the distance between the anterior surface of the crystalline lens and the posterior surface of ICL/TICL (V4c) on the optical axis using the Pentacam].

\section{Statistical Analyses}

All statistical analyses were performed using SPSS 23.0 software (IBM, Armonk, NY, US). The normality of all data was first checked using the Shapiro-Wilk-test. Paired $t$-tests or Wilcoxon signed-rank-tests were used to compare pre- and postoperative data and data between different groups. Pearson's correlation or Spearman's rank correlation were performed to determine the associations between age, spherical equivalence, pre-operative CACD, PACD, ACA, PD, Kf, Ks, corneal posterior radius, astigmatism axis, axial length, WTW, horizontal STS, axis rotation of TICLs, post-operative PACD, ACA, and vault. Multiple stepwise regression analysis was performed to predict post-operative PACD/ACA using significant correlation factors in Pearson's correlation or Spearman's rank correlation analysis as independent variables. $P<0.05$ was considered statistically significant.

\section{RESULTS}

Ninety-two eyes of 46 patients who underwent ICL (V4c) implantation in one eye (Group A, 46 eyes) and an identically sized TICL (V4c) implantation in the contralateral eye (Group B, 46 eyes) were included. Pre-operative demographic data and pre-operative ocular measurements are displayed in Tables 1, 2.

\section{Safety}

All surgeries were uneventful, and no complications occurred during the follow-up period. The safety indices (post-operative
CDVA/pre-operative CDVA) were $1.34 \pm 0.32$ and $1.25 \pm 0.16$ in ICL and TICL groups, respectively. The LogMar CDVA values at the final follow-up were $-0.09 \pm 0.06$ in the ICL group and -0.10 \pm 0.04 in the TICL group. At the final follow-up, no patient lost lines of CDVA; 63.04 and $73.91 \%$ achieved the same CDVA as pre-operatively or increased by one line; and 36.96 and $26.09 \%$ increased by two or more lines in the ICL and TICL groups, respectively (Figure 2A). Safety indices were $1.39 \pm 0.32,1.29 \pm$ $0.17,1.25 \pm 0.17$, and $1.25 \pm 0.16$ in the $12.6 \mathrm{~mm} \mathrm{ICL}, 13.2 \mathrm{~mm}$ ICL, $12.6 \mathrm{~mm}$ TICL, and $13.2 \mathrm{~mm}$ TICL groups, respectively.

No significant differences were found for IOP or ECD between ICL and TICL groups or between subgroups at pre- and postoperative time points, as well as between pre- and post-operative time points in each group $(P>0.05$; Tables 2,3$)$.

\section{Efficacy and Predictability}

The efficacy indices (post-operative UDVA/pre-operative CDVA) were $1.20 \pm 0.24$ and $1.19 \pm 0.19$ in the ICL and TICL groups, respectively. The LogMar UDVA values at the final follow-up were $-0.05 \pm 0.07$ in the ICL group and $-0.07 \pm 0.06$ in the TICL group. At the final follow-up, all eyes had post-operative LogMAR UDVAs of 0.5 or better, and 91.30 and $95.65 \%$ achieved better than LogMAR UDVAs of 0 in the ICL and TICL groups (Figures 2B,C).

Post-operatively, $88.23 \%$ (40 eyes) of the ICL group and $85.29 \%$ (39 eyes) of the TICL group eyes achieved within \pm $0.50 \mathrm{D}$ of the attempted spherical equivalence (SE). All eyes were within $\pm 1.00 \mathrm{D}$ of the attempted SE in both groups (Figures 2D,E); 95.65\% (44 eyes) in the TICL group had postoperative astigmatisms of $\leq 0.50 \mathrm{D}$ (Figure $2 \mathrm{~F}$ ).

\section{PACD and ACA}

After surgery, NPACD decreased by $41.91 \pm 9.08 \%$ and 48.55 $\pm 8.76 \%$ in the ICL and TICL groups, respectively, while TPACD decreased by $42.66 \pm 6.35 \%$ and $50.21 \pm 7.35 \%$, respectively. NACA decreased by $39.42 \pm 7.61 \%$ and $45.97 \pm$ $7.30 \%$, respectively, while TACA decreased by $37.66 \pm 6.82 \%$ and $44.80 \pm 9.69 \%$ in the ICL and TICL groups, respectively. Variations of PACD and ACA of TICL group were significantly greater than those of ICL group. Post-operative PACD and ACA in the TICL group were significantly lower than values in the 


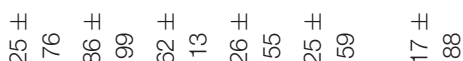

辛

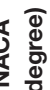

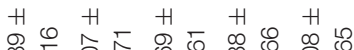

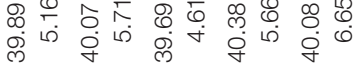

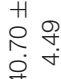

을

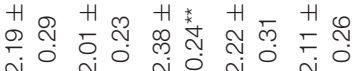

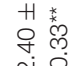

i o

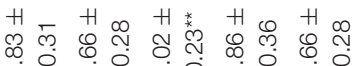

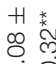

H

品

要

$\begin{array}{llllll}H & H & H & H\end{array}$

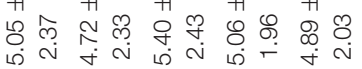

茎

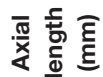

H

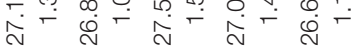

in 8

ลั่

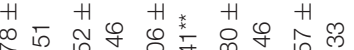

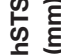

ᄃ.

等

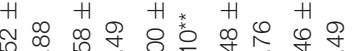

高全

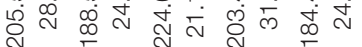

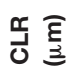

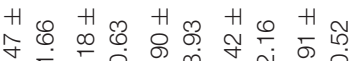

을

ठ্्ల 함

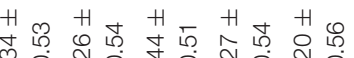

ल⿸户

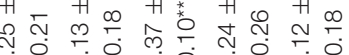

迖

瑝

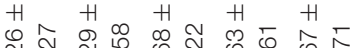

চ

疋 $\overline{\underline{\varepsilon}}$

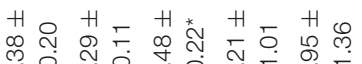

ஸु)

送家

H

$\left.\begin{array}{c}0 \\ N\end{array}\right]$

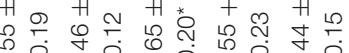

음

อ

$\underline{x}$

อ

$\frac{\overline{5}}{\underline{z}}$

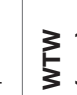

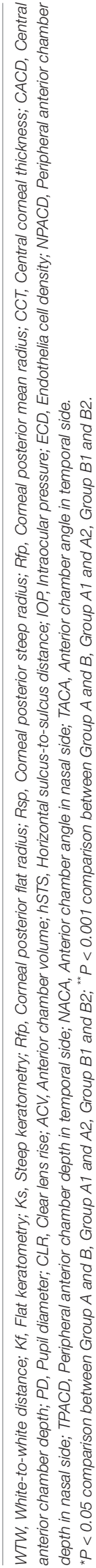

ICL group $(P<0.05$; Table 3). One exemplary case is shown in Figure 3.

There were statistically significant differences in pre- and post-operative PACD and ACA variations between the 12.6 and 13.2 mm ICL/TICL groups $(P<0.05$; Table 3$)$.

Pre-operative PACD, WTW, CACD, cylindrical power of TICL, astigmatism axis, axis rotation of TICLs and post-operative vault correlated significantly with post-operative PACD $(P<$ 0.05). Pre-operative ACA, PD, size of ICL/TICL, cylindrical power of TICL, astigmatism axis, axis rotation of TICLs and post-operative vault correlated significantly with post-operative ACA $(P<0.05$; Table 4). Corneal posterior radius showed no significant correlation with post-operative PACD and ACA in univariate correlation analysis.

Results of the multiple stepwise regression analysis are displayed in Table 5. Factors significantly associated with postoperative NPACD/TPACD included pre-operative CACD and post-operative vault (NPACD adjusted $\mathrm{R}^{2}=0.368$; TPACD adjusted $\mathrm{R}^{2}=0.296$ ). Factors significantly associated with postoperative NACA/TACA included pre-operative NACA/TACA, and post-operative vault (NACA adjusted $\mathrm{R}^{2}=0.665$; TACA adjusted $\mathrm{R}^{2}=0.294$ ).

\section{Iris and Ciliary Body Cysts}

Iris and ciliary body cysts were found in 14 eyes of 8 patients (binocular cysts in 6 patients and monocular cysts in 2 eyes) using UBM examinations. There were nine eyes with a single cyst and five eyes with multiple cysts. All cysts were located in horizontal direction. Post-operative IOP, CACD, and vault were $13.47 \pm 2.21 \mathrm{mmHg}, 2.07 \pm 0.12 \mathrm{~mm}$, and $693.33 \pm 126.59 \mu \mathrm{m}$, respectively. Pre- and post-operative NPACD values were $1.64 \pm$ $0.38 \mathrm{~mm}$ and $0.77 \pm 0.15 \mathrm{~mm}$, pre- and post-operative NACA were $37.07 \pm 4.66^{\circ}$ and $19.13 \pm 3.65^{\circ}$, while pre- and postoperative TPACD were $2.09 \pm 0.22 \mathrm{~mm}$ and $1.11 \pm 0.24 \mathrm{~mm}$, TACA were $41.06 \pm 6.56^{\circ}$ and $23.45 \pm 5.52^{\circ}$, respectively. NACA, TACA, NPACD, TPACD decreased by $48.64 \pm 4.34 \%, 43.04 \pm$ $9.40 \%, 52.93 \pm 4.25 \%$, and $46.67 \pm 9.31 \%$, respectively, compared with pre-operative values. None of the eyes had PAC or high IOP.

\section{DISCUSSION}

The present study was the first consecutive case series and contralateral eye comparison designed to investigate PACD and ACA after ICL and TICL (V4c) implantation. After 1 year of implantation, NPACD, TPACD, NACA, and TACA all decreased significantly in ICL and TICL (V4c) groups. Postoperative PACD and ACA for TICL (V4c) were significantly lower than those for ICL (V4c). Variations of these values for TICL (V4c) were significantly larger than those of ICL (V4c), although pre-operative binocular ocular measurements such as SE, WTW, and CACD remained constant. Vault for TICL (V4c) was significantly higher than that for ICL (V4c). We speculated that the higher vault might push the iris forward, resulting in more changes of anterior chamber structure after TICL (V4c) implantation. Results of correlation analysis and stepwise multivariate regression analysis validated the correlation between PACD/ACA and vault. The rotation of TICL 


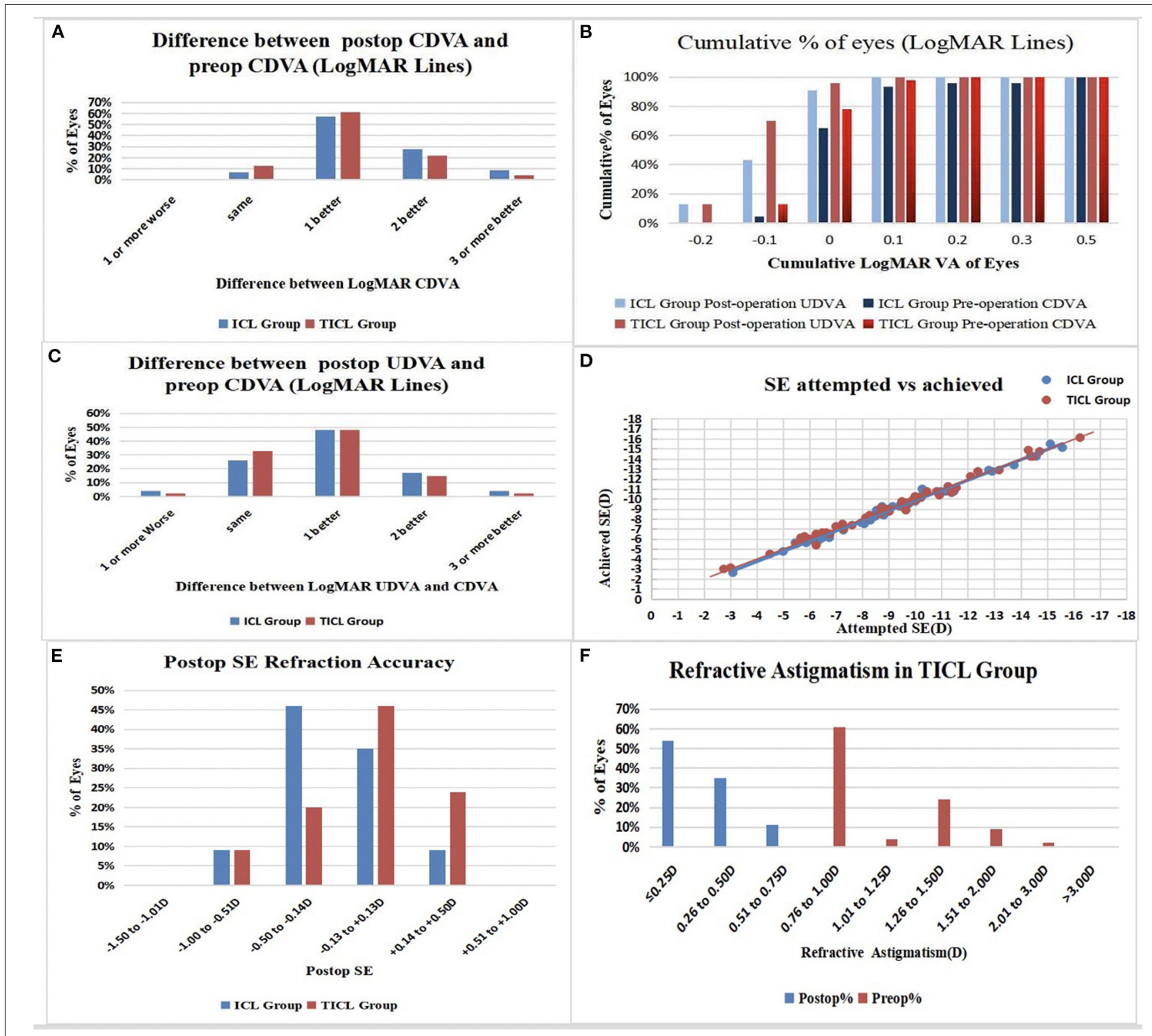

FIGURE 2 | (A) Changes of corrected distance visual acuity (CDVA): postop vs. preop CDVA; (B) Cumulative percentage of the eyes attaining specified cumulative levels of uncorrected distance visual acuity (UDVA); (C) Changes of postop UDVA vs. preop CDVA; (D) Attempted spherical equivalent (SE) refraction change vs. the achieved SE refraction change at the last follow-up were plotted; (E) Distribution of postop SE refraction; (F) Distribution of preop and postop astigmatism amplitude in TICL Group.

(V4c) significantly correlated with post-operative PACD/ACA in univariate correlation analysis, however no significant correlation was found in stepwise multivariate regression analysis in the present study.

Safety of ICL/TICL implantation has been a persistent concern (17-19). PACD and ACA in the ideal range are prerequisites for the safety of post-operative surgery. Zeng et al. (9) reviewed 616 myopic eyes with the previous version of ICL/TICL (V4) implantation and found eight eyes with ICL/TICL exchange for high vault leading to shallow ACD with angle closure in any quadrant or larger PD than pre-operative measurements with severe night glare. Garcia-De la Rosa et al. (20) found significant reductions in the iridocorneal angle after ICL/TICL (V4c) implantation in mesopic, photopic, and scotopic conditions. These findings suggest that it is worth investigating changes of anterior chamber structure after ICL/TICL (V4c) implantation so as to improve surgical quality and safety.

In the present study, the safety and efficacy indices were 1.34 \pm 0.32 and $1.20 \pm 0.24$ for ICL (V4c), and the indices were 1.25 \pm 0.16 and $1.19 \pm 0.19$ for TICL (V4c). Previously, our team reported a post-operative safety index for ICL (V4c) of $1.80 \pm$ 0.89 and an efficacy index of $1.54 \pm 1.07$ (21). The present study 


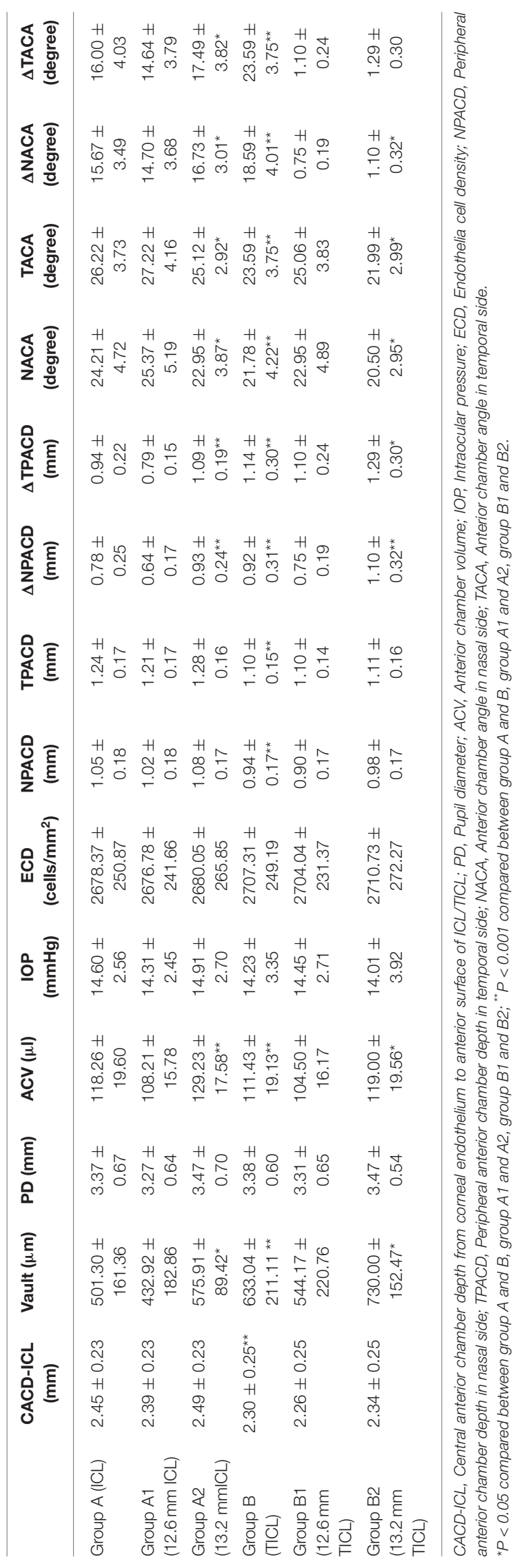

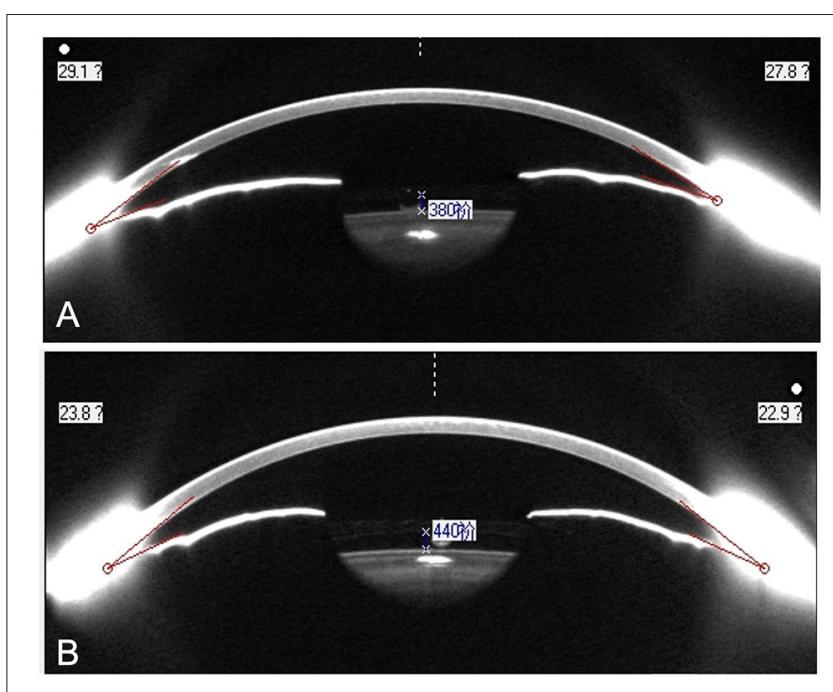

FIGURE 3 | Anterior chamber image of the right eye with $13.2 \mathrm{~mm} \mathrm{ICL} \mathrm{(V4C)}$ (A) and left eye with $13.2 \mathrm{~mm}$ TICL (V4C) (B). Horizontal minimal ACA was $22.2^{\circ}$ in the right eye and $16.4^{\circ}$ in the left eye.

further demonstrated excellent safety and efficacy of variously sized ICL/TICL (V4c). At the final follow-up, 88.23 and $85.29 \%$ of eyes for ICL (V4c) and TICL (V4c), respectively, were within \pm $0.50 \mathrm{D}$ of the attempted SE, while all eyes from both groups were within $\pm 1.00 \mathrm{D}$ in the present study. Garcia-De la Rosa et al. (20) reported that, among 76 eyes implanted with ICL/TICL(V4c), $54 \%$ were within $\pm 0.50 \mathrm{D}$ for average SE and $84 \%$ were within $\pm 1.00 \mathrm{D}$ at 12 months post-operatively. The predictability of SE values in the present study was better than that reported by Garcia-De la Rosa et al., possibly due to the discrepancy of the maximum spherical and astigmatic diopter of eyes (-14.00 DS and $-2.50 \mathrm{DC}$ in the present study vs. $-22.25 \mathrm{DS}$ and $-7.00 \mathrm{DC}$ in Garcia-De la Rosa et al.'s study).

ICL (V4c) was horizontally placed in the ciliary sulcus, and axis rotation of TICL (V4c) was within $13^{\circ}$ in the present study. PACD and ACA significantly decreased after ICL/TICL (V4c) implantation according to Pentacam HR examinations. Our results were similar to those in former reports of postoperative anterior chamber change in response to different types of phakic intraocular lens (IOL) implantation. Javaloy et al. (22) reported that peripherally shallow anterior chamber appeared to be a decisive factor for a $28.78 \%$ decrease of endothelium after iris claw phakic IOL implantation during the 5-year followup. In another study, Benda et al. (23) reported CACD and ACA width decreased 3 years after ICL (ICH V3) implantation compared with pre-operative measurements using Pentacam. Chung et al. (24) reported a $31.8 \%$ trabecular-iris angle (TIA) reduction detected by UBM and a $41.5 \%$ angle opening distance at $500 \mu \mathrm{m}$ from the scleral spur $\left(\mathrm{AOD}_{500}\right)$ decreased at 1 month after ICL (V4) implantation, and no further reduction was observed thereafter in 2-year follow-up. Fernandez-Vigo et al. (25) found significant TIA narrowing of $34.5-42 \%$ and postoperative $\mathrm{AOD}_{500}$ decreasing of $50.3-58.4 \%$ at 3 months postoperatively and no further decrease in 2-year follow-up. Despite 
TABLE 4 | Correlations between post-operative anterior chamber measurements and different ocular factors examined in the study group.

\begin{tabular}{|c|c|c|c|c|c|c|c|c|c|c|}
\hline & \multicolumn{2}{|c|}{ Pre-operative Pre-operative } & \multirow[t]{2}{*}{ CACD } & \multirow[t]{2}{*}{ WTW } & \multirow[t]{2}{*}{ ICL/TICL Size } & \multirow{2}{*}{$\begin{array}{c}\text { Cylindrical } \\
\text { power }\end{array}$} & \multirow{2}{*}{$\begin{array}{l}\text { Astigmatism } \\
\text { axis }\end{array}$} & \multirow{2}{*}{$\begin{array}{l}\text { Axis rotation } \\
\text { of TICL }\end{array}$} & \multirow[t]{2}{*}{ PD } & \multirow[t]{2}{*}{ Vault } \\
\hline & ACA & PACD & & & & & & & & \\
\hline Post-NPACD & / & $0.495^{\star \star}$ & $0.444^{\star \star}$ & $0.306^{*}$ & / & $0.235^{\star}$ & $-0.233^{\star \star}$ & $-0.238^{\star \star}$ & / & $-0.443^{\star \star}$ \\
\hline Post-NACA & $0.638^{\star \star}$ & / & / & / & $-0.209^{\star}$ & $0.257^{\star}$ & $-0.203^{\star \star}$ & $-0.257^{\star \star}$ & $-0.220^{\star}$ & $-0.266^{\star}$ \\
\hline Post-TPACD & / & $0.415^{\star \star}$ & $0.366^{\star \star}$ & $0.264^{*}$ & / & $0.338^{\star}$ & $-0.262^{\star \star}$ & $-0.227^{\star \star}$ & / & $-0.394^{*}$ \\
\hline Post-TACA & $0.402^{\star \star}$ & / & / & / & $-0.303^{\star}$ & $0.306^{*}$ & $-0.307^{\star \star}$ & $-0.288^{\star \star}$ & $-0.304^{\star}$ & $-0.273^{\star}$ \\
\hline
\end{tabular}

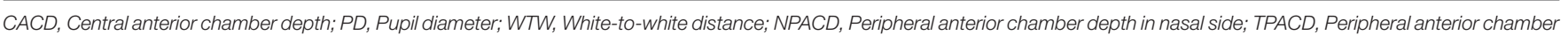
depth in temporal side; NACA, Anterior chamber angle in nasal side; TACA, Anterior chamber angle in temporal side.

${ }^{*} P$-value of correlation coefficient $<0.05$; ${ }^{\star *} P$-value of correlation coefficient $<0.001$.

TABLE 5 | Results of multiple stepwise regression analysis for prediction of post-operative PACD and ACA.

\begin{tabular}{|c|c|c|c|c|}
\hline & Predictors & $\begin{array}{l}\text { Unstandardized } \\
\text { coefficients }\end{array}$ & $\begin{array}{l}\text { Standardized } \\
\text { coefficients }\end{array}$ & $P$-value \\
\hline \multirow[t]{4}{*}{ NPACD } & Constant & -0.417 & & 0.096 \\
\hline & $\begin{array}{l}\text { Pre-operative } \\
\text { CACD }\end{array}$ & 0.482 & 0.626 & 0.000 \\
\hline & Vault & 0.00 & -0.373 & 0.005 \\
\hline & \multicolumn{4}{|c|}{$R^{2}=0.397$, Adjusted $R^{2}=0.368$} \\
\hline \multirow[t]{4}{*}{ TPACD } & Constant & 0.139 & & 0.608 \\
\hline & $\begin{array}{l}\text { Pre-operative } \\
\text { CACD }\end{array}$ & 0.355 & 0.542 & 0.000 \\
\hline & Vault & 0.00 & -0.397 & 0.004 \\
\hline & \multicolumn{4}{|c|}{$R^{2}=0.328$, Adjusted $R^{2}=0.296$} \\
\hline \multirow[t]{4}{*}{ NACA } & Constant & 6.743 & & 0.608 \\
\hline & $\begin{array}{l}\text { Pre-operative } \\
\text { NACA }\end{array}$ & 0.506 & 0.679 & 0.000 \\
\hline & Vault & -0.009 & -0.427 & 0.000 \\
\hline & \multicolumn{4}{|c|}{$R^{2}=0.680$, Adjusted $R^{2}=0.665$} \\
\hline \multirow[t]{4}{*}{ TACA } & Constant & 18.332 & & 0.000 \\
\hline & Vault & -0.008 & -0.471 & 0.001 \\
\hline & $\begin{array}{l}\text { Pre-operative } \\
\text { TACA }\end{array}$ & 0.244 & 0.375 & 0.005 \\
\hline & \multicolumn{4}{|c|}{$R^{2}=0.325$, Adjusted $R^{2}=0.294$} \\
\hline
\end{tabular}

NPACD, Peripheral anterior chamber depth in nasal side; CACD, Central anterior chamber depth; TPACD, Peripheral anterior chamber depth in temporal side; NACA, Anterior chamber angle in nasal side; TACA, Anterior chamber angle in temporal side.

Variables in the table body are ordered according to the strength of the contribution, which was based on the standardized partial regression coefficient.

horizontal placement of ICL, angle narrowing was similar in both horizontal and inferior quadrants. It is speculated that angle narrowing after ICL (V4c) is caused by the pushing forward of iris in the crystal optical area due to the concave optical area on the anterior surface of ICL being larger than the pupil. In the present study, percentage of PACD decreasing and ACA narrowing were similar to $\mathrm{AOD}_{500}$ and TIA decreasing in $\mathrm{V} 4 \mathrm{c}$ study by Fernandez-Vigo (25). Primary angle closure becomes a significant possibility when ACA below $20^{\circ}$ (6). Obviously, postoperative PACD and ACA in the present study decreased within the safety range. In previous UBM and anterior segment optical coherence tomography (AS-OCT) studies, $\mathrm{AOD}_{500}$ decreased by a larger percentage than did TIA. In the present study, postoperative PACD decreased by a larger percentage than did ACA.

In the present study, post-operative ACA for $13.2 \mathrm{~mm}$ lenses was significantly lower than $12.6 \mathrm{~mm}$ lenses, and the change of ACA for $13.2 \mathrm{~mm}$ lenses was larger than that of $12.6 \mathrm{~mm}$ lenses, suggesting a greater effect of larger-sized lenses on ACA. To our knowledge, this is the first report to compare the changes of PACD and ACA between ICL/TICL (V4c) with different sizes (12.6 vs. $13.2 \mathrm{~mm}$ ). Zeng et al. (9) recorded outcomes after implantation of ICL/TICL (V4) of different sizes without central hole $(11.5,12.0$, and $12.5 \mathrm{~mm})$ and found that eyes with larger-sized phakic interocular lenses were more likely to need ICL/TICL exchange for excessively high vault, resulting in ACA closure in any quadrant. In the present study, the mean vault of $13.2 \mathrm{~mm}$ lenses was significantly higher than that of $12.6 \mathrm{~mm}$ lenses, which was within the safe range. WTW and CACD are the most important factors in determining the ICL/TICL (V4c) size using the Staar calculator software; consequentially, larger-sized lenses would be selected for eyes with larger WTW and deeper CACD. No significant difference was found in pre-operative ACA among the different subgroups, although the values of WTW, CACD, STS, and ACV in the $13.2 \mathrm{~mm}$ groups were significantly larger than those of the $12.6 \mathrm{~mm}$ ICL/TICL (V4c) groups. These findings suggest that ACA should be taken into consideration while selecting larger-sized V4c lenses.

In this study, stepwise multiple regression analysis revealed that pre-operative CACD and post-operative vault were significantly associated with post-operative PACD. Pre-operative ACA, and post-operative vault were significantly associated with post-operative ACA. Fernandez-Vigo et al. (25) identified pre-operative TIA, age, SE, CACD, axial length, and WTW as predictors of TIA at 1-month post-ICL (V4c) implantation using Fourier-domain OCT (FD-OCT). In a subsequent 2-year follow-up report, Fernandez-Vigo (26) identified pre-operative TIA, age, sex, SE, IOL size, iris thickness at the perpendicular point $500 \mu \mathrm{m}$ to the scleral spur, and WTW affected postoperative TIA. Lee et al. (27) reported that ICL size, STS, age, and mean $\mathrm{K}$ readings were determinants of post-operative vault. In addition to pre-operative ocular measurements, post-operative vault was identified as an important determinant of both postoperative PACD and ACA in the present study, respectively, indicating higher vault might push ICL/TICLs more forwardly and resulting in narrower ACA or shallower PACD. In 2010, Lindland et al. (28) first reported that the vault after toric ICL 
(TICL) (V4 model) implantation was higher than that of ICL (V4 model) and they presumed that addition of a cylindrical lens to the TICL optic zone may contribute to vault difference. In another study conducted by our team, we investigated the reason of ICL/TICL vault difference using multivariate parameters statistical processing and found that cylindrical power of TICL might contribute to this difference (29). We hope our conclusion will make some significance in ICL/TICL implantation surgery. Although different equipment were applied, the present study of PACD and ACA measured by Pentacam showed similar results as those of the FD-OCT study of TIA and $\mathrm{AOD}_{500}$ in Fernandez-Vigo's reports, suggesting that changes of anterior chamber parameters could be predicted based on pre-operative ocular measurements $(25,26)$.

Post-operative NPACD, TPACD, NACA, and TACA were lower than pre-operative measurements in eyes with iris and/or ciliary body cysts in the present study. Li and associates (30) found no significant differences in post-operative $\mathrm{AOD}_{500}$ and TIA between eyes with and without cysts. In Li's study, cysts were found predominantly in the inferior and temporal quadrants, while cysts were primarily located in the horizontal direction in the present study. Nevertheless, further research on relationships of cysts' location, size, ICL/TICL placement and PACD, and ACA change is of great importance to improve safety of ICL/TICL implantation.

The current study has some limitations. First, it utilized a relatively small sample size for few numbers of patients satisfying contralateral eye comparison design. Second, only two sizes of ICL/TICL (V4c) were analyzed, as 12.6 and $13.2 \mathrm{~mm}$ ICL/TICL (V4c) were selected frequently for these included patients. Future studies should be conducted with lager sample size and various lens sizes so as to make a comprehensive comparison.

In conclusion, it is possible to measure PACD and ACA using the Pentacam in ICL/TICL (V4c) implanted eyes. Preand post-operative variations of PACD and ACA were greater in eyes after TICL (V4c) implantation than with ICL (V4c) implantation and implantations of larger-sized lenses compared with smaller lenses. Post-operative PACD and ACA correlated with pre-operative anterior chamber structure and vault.

\section{REFERENCES}

1. Siedlecki J, Schmelter V, Mayer WJ, Schworm B, Priglinger SG, Dirisamer $\mathrm{M}$, et al. SMILE versus implantable collamer lens implantation for high myopia: a matched comparative study. J Refract Surg. (2020) 36:150-9. doi: 10.3928/1081597X-20200210-02

2. Sari E, Pinero D, Kubaloglu A, Evcili P, Koytak A, Kutluturk I, et al. Toric implantable collamer lens for moderate to high myopic astigmatism: 3-year follow-up. Graefes Arch Clin Exp Ophthalmol. (2013) 251:1413-22. doi: 10.1007/s00417-012-2172-8

3. Sanders DR, Doney K, Poco M. United States food and drug administration clinical trial of the implantable collamer lens (ICL) for moderate to high myopia: three-year follow-up. Ophthalmology. (2004) 111:1683-92. doi: 10.1016/j.ophtha.2004. 03.026

4. Elmohamady MN, Abdelghaffar W. Anterior chamber changes after implantable collamer lens implantation in high myopia using

\section{DATA AVAILABILITY STATEMENT}

The raw data supporting the conclusions of this article will be made available by the authors, without undue reservation.

\section{ETHICS STATEMENT}

The studies involving human participants were reviewed and approved by the institutional ethics board of Eye and ENT Hospital of Fudan University (No. 2013015-1) and informed consent was taken from all the patients after a complete description of the study. The patients/participants provided their written informed consent to participate in this study.

\section{AUTHOR CONTRIBUTIONS}

The study concept and design were formulated by JiaZ, JinZ, and XZ. Data collection was done by JiaZ, JinZ, WY, HM, LN, JS, XW, and $X Z$. Analysis and interpretation of data was undertaken by JiaZ, JinZ, and XZ. Drafting of the manuscript was carried out by JiaZ, JinZ, and XZ. Critical revision of the manuscript was done by JiaZ, JinZ, and XZ. Supervision was done by XZ. All authors contributed to the article and approved the submitted version.

\section{FUNDING}

This work was supported in part by the National Natural Science Foundation of China (Grant No. 81770955); Joint research project of new frontier technology in municipal hospitals (SHDC12018103); the Project of Shanghai Science and Technology (Grant No. 17411950200); the National Natural Science Foundation of China for Young Scholars (Grant No. 81600762); the Project of Shanghai Science and Technology (Grant No. 19140900700) and the Shanghai Shenkang Hospital Development Center (Grant No. SHDC12016207).

\section{ACKNOWLEDGMENTS}

We would like to thank Editage (www.editage.cn) for English language editing.

Pentacam: a prospective study. Ophthalmol Ther. (2017) 6:343-9. doi: 10.1007/s40123-017-0109-3

5. Foo L-L, Nongpiur ME, Allen JC, Perera SA, Friedman DS, He M, et al. Determinants of angle width in chinese singaporeans. Ophthalmology. (2012) 119:278-82. doi: 10.1016/j.ophtha.2011.07.049

6. Foster PJ, Aung T, Nolan WP, Machin D, Baasanhu J, Khaw PT, et al. Defining "occludable" angles in population surveys: drainage angle width, peripheral anterior synechiae, and glaucomatous optic neuropathy in east asian people. $\mathrm{Br}$ J Ophthalmol. (2004) 88:486-90. doi: 10.1136/bjo.2003. 020016

7. Shaffer RN. A suggested anatomic classification to define the pupillary block glaucomas. Invest Ophthalmol. (1973) 12:540-2.

8. Wong H-T, Chua JLL, Sakata LM, Wong MHY, Aung HT, Aung T. Comparison of slitlamp optical coherence tomography and scanning peripheral anterior chamber depth analyzer to evaluate angle closure in asian eyes. Arch Ophthalmol. (2009) 127:599-603. doi: 10.1001/archophthalmol. 2009.41 
9. Zeng Q-Y, Xie X-L, Chen Q. Prevention and management of collagen copolymer phakic intraocular lens exchange: causes and surgical techniques. $J$ Cataract Refract Surg. (2015) 41:576-84. doi: 10.1016/j.jcrs.2014.06.036

10. Sanders DR, Vukich JA, Doney K, Gaston M. U.S. food and drug administration clinical trial of the implantable contact lens for moderate to high myopia. Ophthalmology. (2003) 2:255-66. doi: 10.1016/S0161-6420(02)01771-2

11. Friedman DS, Gazzard G, Foster P, Devereux J, Seah S. Ultrasonographic biomicroscopy, scheimpflug photography, and novel provocative tests in contralateral eyes of chinese patients initially seen with acute angle closure. Arch Ophthalmol. (2003) 121:633-42. doi: 10.1001/archopht.121.5.633

12. Wojciechowski R, Congdon N, Anninger W, Broman AT. Age, gender, biometry, refractive error, and the anterior chamber angle among alaskan eskimos. Ophthalmology. (2003) 110:365-75. doi: 10.1016/S0161-6420(02)01748-7

13. Kurita N, Mayama C, Tomidokoro A, Aihara M, Araie M. Potential of the pentacam in screening for primary angle closure and primary angle closure suspect. J Glaucoma. (2009) 18:506-12. doi: 10.1097/IJG.0b013e318193c141

14. Smith S, Singh K, Lin S, Chen P, Chen T, Francis B, et al. Evaluation of the anterior chamber angle in glaucoma: a report by the american academy of ophthalmology. Ophthalmology. (2013) 120:1985-97. doi: 10.1016/j.ophtha.2013.05.034

15. Eissa SA, Sadek SH, El-Deeb MWA. Anterior chamber angle evaluation following phakic posterior chamber collamer lens with CentraFLOW and its correlation with ICL vault and intraocular pressure. J Ophthalmol. (2016) 2016:1-7. doi: 10.1155/2016/7012826

16. Zhao J, Luo D, Sun Y, Niu L, Zhao F, Wang X, et al. Implanting a posterior chamber phakic intraocular lens in highly myopic eyes with peripheral primary iris and ciliary body cysts. Eur J Ophthalmol. (2019) 29:171-7. doi: $10.1177 / 1120672118766445$

17. Jiménez-Alfaro I, Benítez del Castillo JM, García-Feijoó J, Gil de Bernabé JG, Serrano de La Iglesia JM. Safety of posterior chamber phakic intraocular lenses for the correction of high myopia. Ophthalmology. (2001) 108:90-9. doi: 10.1016/S0161-6420(00)00403-6

18. Higueras-Esteban A, Ortiz-Gomariz A, Gutierrez-Ortega R, VillaCollar C, Abad-Montes J, Fernandes P, et al. Intraocular pressure after implantation of the visian implantable collamer lens with centra flow without iridotomy. Am J Ophthalmol. (2013) 156:800-5. doi: 10.1016/j.ajo.2013. 05.018

19. Packer M. Meta-analysis and review: effectiveness, safety, and central port design of the intraocular collamer lens. Clin Ophthalmol. (2016) 10:1059-77. doi: 10.2147/OPTH.S111620

20. Garcia-De la Rosa G, Olivo-Payne A, Serna-Ojeda JC, Salazar-Ramos MS, Lichtinger A, Gomez-Bastar A, et al. Anterior segment optical coherence tomography angle and vault analysis after toric and non-toric implantable collamer lens V4c implantation in patients with high myopia. Br J Ophthalmol. (2018) 102:544-8. doi: 10.1136/bjophthalmol-2017-310518

21. Chen X, Guo L, Han T, Wu L, Wang X, Zhou X. Contralateral eye comparison of the long-term visual quality and stability between implantable collamer lens and laser refractive surgery for myopia. Acta Ophthalmol. (2019) 97:e471-8. doi: 10.1111/aos.13846

22. Javaloy J, Javaloy T, Borras F, Vidal MT, Mulet E, Belda JI, et al. Safety of iris claw phakic intraocular lenses: an endothelial microscopy long term study. Invest Ophthalmol Vis Sci. (2004) 45:337. doi: 10.1167/iovs.03-0693

23. Benda F, Filipová L, Filipec M. Correction of moderate to high hyperopia with an implantable collamer lens: medium-term results. J Refract Surg. (2014) 30:526-533. doi: 10.3928/1081597X-20140711-05

24. Chung T-Y, Park SC, Lee MO, Ahn K, Chung E-S. Changes in iridocorneal angle structure and trabecular pigmentation with STAAR implantable collamer lens during 2 years. J Refract Surg. (2009) 25:251-8. doi: 10.3928/1081597X-20090301-03

25. Fernández-Vigo J, Macarro-Merino A, Fernández-Vigo C, FernándezVigo J, Martnez-de-la-Casa J, Fernández-Pérez C. Effects of implantable collamer lens $\mathrm{v} 4 \mathrm{c}$ placement on iridocorneal angle measurements by fourierdomain optical coherence tomography. Am J Ophthalmol. (2016) 162:43-52. doi: 10.1016/j.ajo.2015.11.010

26. Fernández-Vigo JI, Macarro-Merino A, Fernández-Vigo C, FernándezVigo JÁ, Pablo-Gómez-de-Liaño L, Fernández-Pérez C, et al. Impacts of implantable collamer lens $\mathrm{v} 4 \mathrm{c}$ placement on angle measurements made by optical coherence tomography: two-year follow up. Am J Ophthalmol. (2017) 181:37-45. doi: 10.1016/j.ajo.2017.06.018

27. Lee D-H, Choi S-H, Chung E-S, Chung T-Y. Correlation between preoperative biometry and posterior chamber phakic visian implantable collamer lens vaulting. Ophthalmology. (2012) 119:272-7. doi: 10.1016/j.ophtha.2011. 07.047

28. Lindland A, Heger H, Kugelberg M, Zetterström C. Vaulting of myopic and toric implantable collamer lenses during accommodation measured with visante optical coherence tomography. Ophthalmology. (2010) 117:1245-50. doi: 10.1016/j.ophtha.2009.10.033

29. Zhao J, Zhao J, Yang W, Li M, Hao G, Chen Z, et al. Consecutive contralateral comparison of toric and non-toric implantable collamer lenses V4c in vault after implantation for myopia and astigmatism. Acta Ophthalmologica. (2020) doi: 10.1111/aos.14720

30. Li Z, Xu Z, Wang Y, Liu Q, Chen B. Implantable collamer lens surgery in patients with primary iris and/or ciliary body cysts. BMC Ophthalmol. (2018) 18:287. doi: 10.1186/s12886-018-0935-7

Conflict of Interest: The authors declare that the research was conducted in the absence of any commercial or financial relationships that could be construed as a potential conflict of interest.

Copyright (c) 2021 Zhao, Zhao, Yang, Miao, Niu, Shang, Wang and Zhou. This is an open-access article distributed under the terms of the Creative Commons Attribution License (CC BY). The use, distribution or reproduction in other forums is permitted, provided the original author(s) and the copyright owner(s) are credited and that the original publication in this journal is cited, in accordance with accepted academic practice. No use, distribution or reproduction is permitted which does not comply with these terms. 